\title{
Identifying Persons Who Inject Drugs in Medical Examiner Data in Maricopa County, AZ
}

\author{
Rasneet S. Kumar ${ }^{1}$, Kaitlyn Sykes ${ }^{1,}$ 3, Kate Goodin ${ }^{1}$, Brian Hanratty ${ }^{1}$ 2 , Jessica R. White $^{1}$ \\ ${ }^{1}$ Office of Epidemiology, Maricopa County Department of Public Health, Phoenix, Arizona, United States, ${ }^{2}$ Bioinformatics Core Facility, Biodesign \\ Institute, Arizona State University, Tempe, Arizona, United States, ${ }^{3}$ CSTE Applied Epidemiology Fellowship, Atlanta, Georgia, United States
}

\section{Objective}

To determine whether data from the Office of the Medical Examiner are useful for conducting injection drug use surveillance in Maricopa County, Arizona, and to describe the characteristics of decedents who died from a drug overdose, were investigated by the county's medical examiner, and had an indication of injection drug use.

\section{Introduction}

The rate of drug overdose deaths in the United States has increased steadily since 2000. Injection drug use, a practice associated with infectious disease transmission, has likely increased along with this upward trend in drug overdoses. Injection drug use surveillance is difficult to conduct at a public health department because there are no specific Internal Classification of Diseases codes to identify this risk behavior in hospital discharge or vital registration data.

Maricopa County Department of Public Health Office of Epidemiology aimed to identify indications of injection drug use within data from the Office of the Medical Examiner

\section{Methods}

The Office of Epidemiology receives toxicology results for deaths which were ruled by the Maricopa County's medical examiner as substance use related, also referred to as overdose deaths. We explored data from decedents who were investigated by the Maricopa County's medical examiner between 7/1/2016-6/30/2017, had a cause of death from a drug overdose, and screened positive for at least one commonly injected drug (i.e., opioids - general, heroin, methamphetamine, or cocaine). To narrow our search for indications of injection drug use, we requested preliminary investigation reports and medical examiner reports for a random sample of reports (10\% from each drug class) from the Office of the Medical Examiner. Preliminary investigation reports, produced by a scene investigator, included the decedent's medical history, risk factors, circumstances of death, circumstances of death discovery, and scene characteristics. Medical examiner reports included toxicology screen results, autopsy findings, and the cause of death. The Office of the Medical Examiner provided these reports to the Office of Epidemiology in portable document format (PDF) for this analysis. We built a query to identify keywords related to injection drug use (e.g. inject, syringe, needle) and injection injuries (e.g. cellulitis, abscess). We used PDF XChangeViewer's Optical Character Recognition (OCR) function to convert the PDF reports to text data and used Python's String and Collections modules to parse text data for occurrences of keywords within the reports. Reports that included at least one keyword were manually reviewed and classified as probable or ruled out for injection drug use, and characteristics of those with a probable indication of injection drug use were described.

\section{Results}

During the period of interest, 1,127 deaths were caused by drug overdose, of which 930 decedents screened positive for opioids $(\mathrm{N}=673,59.7 \%$ of drug overdose deaths), heroin $(358,38.5 \%)$, methamphetamine $(445,39.5 \%)$, and/or cocaine $(100,11 \%)$. Indications of injection drug use were identified within $48(32.7 \%)$ of the 147 preliminary investigation and medical examiner reports that were reviewed (Table). Common indicators of injection drug use included: history of drug use as a reported risk factor; presence of prescription drugs, illicit drugs, or drug paraphernalia at the scene; body position at the scene; and injuries associated with needle use identified during the autopsy. The most common terms that indicated injection drug use were "syringe", "intravenous", "needle", and "inject". Among persons who had an indication of injection drug use, $85.4 \%$ were male, $52.1 \%$ were between the ages of 20 and 39 years, and $85.4 \%$ were white. 


\section{Conclusions}

Data from the Office of the Medical Examiner's preliminary investigation and medical examiner reports provided rich context for understating the underlying risk factors and circumstances that contributed to drug overdose deaths. Injection drug use paraphernalia found at the scene of death and injuries found during autopsy were well-documented in these reports, which helped us quantify the proportion of decedents who died from overdose that may have injected drugs, by drug class. We were able to describe basic characteristics of this sample, which were consistent with previously published reports describing people who inject drugs. This surveillance method has limitations, however. Decedents reviewed by a medical examiner represent a subset of the drug using population, and findings may not be generalized to the full population. Other data sources and analytical methods must be employed to accurately estimate the number of people who inject drugs in Maricopa County and to describe their characteristics and experiences.

\section{Acknowledgement}

This report was supported in part by an appointment to the Applied Epidemiology Fellowship Program administered by the Council of State and Territorial Epidemiologists (CSTE) and funded by the Centers for Disease Control and Prevention (CDC) Cooperative Agreement Number 1U38OT000143-04

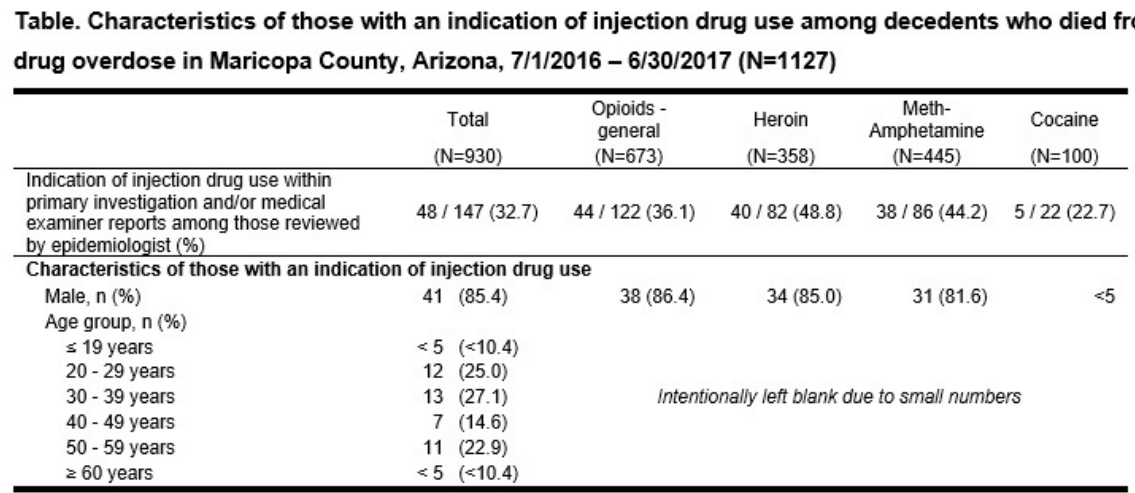

ISDS Annual Conference Proceedings 2019. This is an Open Access article distributed under the terms of the Creative Commons AttributionNoncommercial 4.0 Unported License (http://creativecommons.org/licenses/by-nc/3.0/), permitting all non-commercial use, distribution, and reproduction in any medium, provided the original work is properly cited. 